\title{
Abstract \\ A Computational Study to Identify Some Potential Inhibitors of SARS-CoV-2 Main Protease from Biological Active Quinolones ${ }^{\dagger}$
}

\author{
Lucia Pintilie (1)
}

check for updates

Citation: Pintilie, L. A

Computational Study to Identify

Some Potential Inhibitors of

SARS-CoV-2 Main Protease from Biological Active Quinolones. Chem Proc. 2022, 7, 10. https://doi.org/ $10.3390 /$ chemproc2022007010

Academic Editors: Mihaela Doni, Florin Oancea, Zina Vuluga and Radu Claudiu Fierăscu

Published: 28 February 2022

Publisher's Note: MDPI stays neutral with regard to jurisdictional claims in published maps and institutional affiliations.

Copyright: (C) 2022 by the author. Licensee MDPI, Basel, Switzerland. This article is an open access article distributed under the terms and conditions of the Creative Commons Attribution (CC BY) license (https:// creativecommons.org/licenses/by/ $4.0 /)$.
National Institute for Chemical, Pharmaceutical Research and Development (ICCF), 112 Vitan Av., 031299 Bucharest, Romania; lucia.pintilie@gmail.com

† Presented at the 17th International Symposium "Priorities of Chemistry for a Sustainable Development" PRIOCHEM, Bucharest, Romania, 27-29 October 2021.

Keywords: SARS-CoV-2; drug design; molecular docking; antimicrobial quinolones; antitumoral quinolones

The coronavirus pandemic, known as COVID-19, is caused by severe acute respiratory syndrome coronavirus 2 (SARS-CoV-2). This is a highly pathogenic human coronavirus $(\mathrm{CoV})$ first reported in Wuhan, China [1]. So far, there is no specific treatment available for COVID-19 [2]. The discovery of new antiviral agents is extremely important. For the development of the anti-SARS-CoV-2 drugs, the fastest way is to find potential molecules from the marketed drugs by molecular docking studies [3]. A computational study to identify some potential inhibitors of SARS-CoV-2 main protease from antibacterial and antitumoral quinolones has been realized. Molecular docking studies have been performed to identify and visualize the most likely interaction of the ligand with the protein/enzyme receptor. The docking score and hydrogen bonds formed with the amino acids from of the group interaction atoms are used to predict the binding modes, the binding affinity, and the orientation of the docked ligands in the active site of the protein/enzyme receptor. The docking study have been carried out with some quinolones against the SARS-CoV-2 main protease (PD ID: 5R7Z). The docking study have been carried out with eight 1-ethyl-quinolone compounds with antimicrobial activity and with four 1-benzyl-quinolone compounds with antitumoral activity against the SARS-CoV-2 main protease (PD ID: 5R7Z). The study has been realized relating to two fluoroquinolone compounds known in medical therapeutics: ciprofloxacin and moxifloxacin. The docking studies reveals that all compounds presented good docking score. The best score docking was obtained for 1-benzyl-quinolone compounds. 6FPQ11compound presents the great docking score (Figure 1). From the 1-ethyl-quinolone compounds, FPQ51 compound has the better docking score (Figure 2) in comparison with ciprofloxacin (Score: -54.29; RMSD $0.59 \AA$ ) and with moxifloxacin (Score: -51.53; RMSD $0.50 \AA$ ).

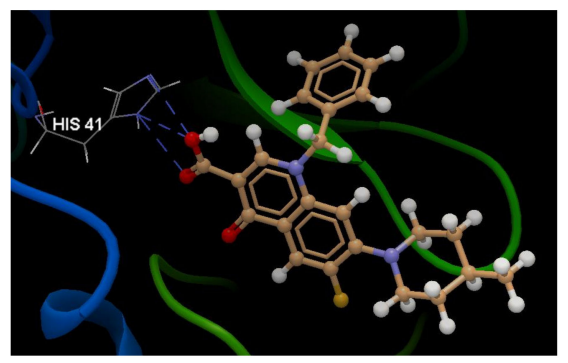

Figure 1. Docking pose of the 6FPQ11 ligand interacting with amino acid residues (Score -66.38; RSMD 0.02). 


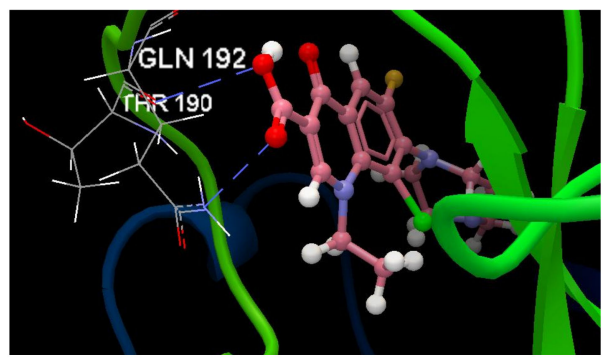

Figure 2. Docking pose of the FPQ51 ligand interacting with amino acid residues (Score -56.28; RMSD 0.62).

Funding: This paper has been financed through the NUCLEU Program, which is implemented with the support of MCID, project no. PN 19-41 0102.

Data Availability Statement: Not applicable.

Conflicts of Interest: The authors declare no conflict of interest.

\section{References}

1. Mohapatra, R.K.; Pintilie, L.; Ashish, K.; Sarangi, A.K.; Das, D.; Sahu, R.; Perekhoda, L. The recent challenges of highly contagious COVID-19; causing respiratory infections: Symptoms, diagnosis, transmission and possible vaccines. Chem. Biol. Drug Des. 2020, 96, 1187-1208. [CrossRef] [PubMed]

2. Marciniec, K.; Beberok, A.; Pęcak, P.; Boryczka, S.; Wrześniok, D. Ciprofloxacin and moxifloxacin could interact with SARS-CoV-2 protease: Preliminary in silico analysis. Pharmacol. Rep. 2020, 72, 1553-1561. [CrossRef] [PubMed]

3. Yacouba, A.; Olowo-okere, A.; Yunusa, I. Repurposing of antibiotics for clinical management of COVID-19: A narrative review. Ann. Clin. Microbiol. Antimicrob. 2021, 20, 37. [CrossRef] [PubMed] 